\title{
Factores asociados al rendimiento académico de los estudiantes del centro de estudios preuniversitarios de la Universidad Nacional Agraria La Molina
}

\author{
Factors associated with academic performance of students in the pre-university center of \\ Universidad Nacional Agraria La Molina
}

\section{Alfredo Moreno LI. ${ }^{1}$; Leandro Huanca V. ${ }^{2}$}

\begin{abstract}
Resumen
El propósito de este estudio fue identificar los factores asociados al rendimiento académico de los estudiantes del Centro de Estudios Preuniversitarios de la Universidad Nacional Agraria La Molina - UNALM, del ciclo del 2011-I, en los cursos de Razonamiento Verbal, Razonamiento Matemático, Algebra, Aritmética, Geometría, Trigonometría, Física, Química y Biología. De acuerdo con los resultados estadísticos del modelo multinivel, se encontró que el tipo de matrícula en el Centro de Estudios Preuniversitarios (CEPRE-UNALM) se encuentra asociado al rendimiento de todos los cursos que se desarrollan en el Centro; el género se encuentra vinculado al rendimiento en Geometría; el número de ciclos se encuentra relacionado con el rendimiento en todos los cursos menos en el de Razonamiento Verbal; el colegio de procedencia se encuentra asociado al rendimiento de los cursos de Aritmética, Razonamiento Verbal y Razonamiento Matemático y el tipo de profesor en el aula se encuentra vinculado al rendimiento de los cursos de Física, Algebra, Química y Biología.
\end{abstract}

Palabras clave: rendimiento estudiantil; investigación educativa; modelos multinivel.

\begin{abstract}
The purpose of this study was to identify the factors associated with academic performance of students in Pre University Studies Center of the UNALM during the 2011-I term on the following subjects: Verbal Reasoning, Mathematical Reasoning, Algebra, Arithmetic, Geometry, Trigonometry, Physics, Chemistry and Biology. According to the statistical results of the multilevel model, it was found that the type of enrollment in CEPRE-UNALM is associated with the performance of all courses offered in the CEPRE-UNALM, gender is associated with the performance in Geometry, number of cycles in the CEPRE-UNALM is associated with the performance of all courses except Verbal Reasoning, school of origin is associated with the performance of Arithmetic, Verbal Reasoning and Mathematical Reasoning and the type of teacher is associated with the performance of Physics, Algebra, Chemistry and Biology.
\end{abstract}

Keywords: student achievement; educational research; multilevel models.

\section{Introducción}

El rendimiento académico de un estudiante depende de muchos factores individuales (edad, lengua materna nativa, género, lugar de procedencia, antecedentes académicos, etc.); familiares (nivel educativo de los padres, nivel socioeconómico y cultural de la familia, etc.) y contextuales (características institucionales y sociodemográficas, procesos para la enseñanza, clima institucional, etc.) que no actúan aisladamente, sino que se interrelacionan e inciden en el rendimiento académico.

De esta manera, se tiene interés en buscar diferentes factores que condicionen el rendimiento académico de los estudiantes, en busca de aportar datos útiles que orienten a la eficiencia del sistema educativo de una institución. Sin embargo, la responsabilidad institucional es clave para evaluar lo que se entiende por rendimiento. Más allá de las condiciones internas de las instituciones y de las prácticas docentes, resulta imprescindible también conocer las características que aportan quienes son los receptores de la labor docente.

Diversas características de los estudiantes han sido consideradas para relacionarlas con el rendimiento académico, desde las características actitudinales, intelectuales y de personalidad del estudiante hasta sus

\footnotetext{
${ }^{1}$ Profesor Asociado de la Facultad de Ciencias. Universidad Nacional Agraria La Molina, Lima, Perú. Email: amoreno@lamolina.edu.pe

${ }^{2}$ Profesor Principal de la Facultad de Ciencias. Universidad Nacional Agraria La Molina, Lima, Perú. Email: 1huanca@lamolina.edu.pe
} 
aspectos motivacionales y de percepción personal. Otros autores han estudiado cómo la pertenencia a un cierto sector socioeconómico o las características personales del estudiante, tales como edad, género y lugar de procedencia, pueden explicar el rendimiento académico. Este constituye un indicador del nivel de aprendizaje alcanzado por el estudiante, representa el nivel de eficacia en la consecución de los objetivos curriculares para las diversas asignaturas y, en nuestro país, se expresa mediante un calificativo o promedio ponderado basado en el sistema vigesimal; es decir, las notas que varían de 0 a 20 puntos, donde el puntaje de 10 o menos es reprobatorio. En el sistema educativo nacional e internacional se da importancia a este indicador y por ello las instituciones educativas necesitan estudiar los determinantes del rendimiento académico de los estudiantes para mejorar sus estrategias de aprendizaje.

El presente trabajo de investigación tiene como propósito identificar los factores asociados al rendimiento académico de los estudiantes del CEPRE-UNALM del ciclo 2011-I, en los nueve cursos que se desarrollan. Para ello, se ha utilizado el análisis multinivel debido a que considera datos que tienen una estructura jerárquica.

\section{Materiales y métodos}

\section{Marco teórico}

El rendimiento académico es entendido por Pizarro (1985) como una medida de las capacidades correspondientes o indicativas que manifiestan en forma estimativa, lo que una persona ha aprendido como consecuencia de un proceso de instrucción o formación. El autor también define el rendimiento desde la perspectiva del estudiante como su capacidad de respuesta frente a estímulos educativos, susceptible de ser interpretado según objetivos o propósitos educativos pre-establecidos. Algunas otras definiciones de rendimiento académico son las siguientes:

El rendimiento académico puede ser entendido en relación a un grupo social que fija unos rangos sobre los niveles mínimos de aprobación y máximos de desaprobación ante un determinado cúmulo de conocimientos y/o aptitudes (Carrasco, 1985).

En forma operativa y tácita se define afirmando que "El rendimiento escolar previo es definido como el número de veces que el alumno ha repetido uno o más cursos." (Heran y Villarroel, 1987).

El rendimiento académico depende, en mayor o menor grado, de numerosas variables que configuran una compleja red en la que es demasiado complejo calibrar la incidencia específica de cada una (Martínez, 2002).

Mizala y Romaguera (2002), analizan los factores que inciden en el rendimiento escolar en Bolivia, identificando de esta manera las variables que intervienen en el análisis de factores asociados que se agrupan en las siguientes categorías:

Variables individuales: características sociales, socioeconómicas y culturales de la familia, trabajo, distancia de la escuela al centro educativo, actitudes e historia educativa (antecedentes individuales del alumno).
Variables escolares: características sociales e institucionales del centro educativo, infraestructura del aula y del centro educativo, composición socioeconómica del aula, clima institucional, características personales y profesionales de los docentes, recursos pedagógicos y cobertura curricular.

\section{Antecedentes}

Los primeros esfuerzos por detectar relaciones estadísticas entre el aprendizaje, el hogar y la escuela utilizaron un método de agregación de variables, concluyendo que el hogar ejercía, en los Estados Unidos, la principal influencia sobre los resultados de las pruebas escolares y que el efecto de la escuela era menor (Coleman et al., 1966).

En 1968, Bowles y Levin presentaron un estudio que colocaba en entredicho las conclusiones del Informe Coleman. Estos autores demostraron que las técnicas de incorporación progresiva de variables habían sido utilizadas sin considerar la existencia de una alta correlación entre las variables representativas del hogar y de la escuela. En el mismo estudio los autores presentan una función de producción de tipo lineal multivariada $\mathrm{y}$, utilizando los mismos datos que utilizó el Informe Coleman, demuestran que la escuela posee, en Estados Unidos, un efecto positivo y significativo sobre el aprendizaje.

El Ministerio de Educación de Bolivia (1997), en sus publicaciones sobre Reforma Educativa, indica la percepción de los directores y profesores sobre los factores que inciden en el rendimiento académico escolar. Los directores concluyen que los factores que favorecen la calidad de la educación son el apoyo familiar, la formación del profesor, el material didáctico apropiado, el currículo acorde con la realidad, la infraestructura adecuada y la administración eficaz; mientras que los aspectos que desfavorecen la calidad de la educación son la situación económica de la familia, la falta de materiales educativos, el salario de los profesores, el tiempo que los estudiantes ven televisión, el aprendizaje en lengua no materna y las enfermedades de los alumnos.

La Unidad de Medición de la Calidad Educativa del Ministerio de Educación del Perú (2004) ha llevado a cabo cuatro evaluaciones nacionales del rendimiento: CRECER 1996, CRECER 1998, la Evaluación Nacional 2001 y la Evaluación Nacional 2004. En los resultados de la Evaluación Nacional 2001 sobre los factores asociados al rendimiento estudiantil, se encontró que existen diferencias en el rendimiento en matemáticas a favor de los estudiantes masculinos respecto de sus pares femeninos. Respecto a las características institucionales, se observó que en el efecto de la variable gestión de dependencia (estatal/ privado), los centros educativos secundarios de gestión privada obtienen resultados superiores a los del sector estatal.

Porto y Di Gresia (2004), en su trabajo sobre la dinámica del desempeño académico, usa un modelo de regresión múltiple y toma, como variable dependiente, la cantidad de materias aprobadas durante cierto período y, como variables explicativas, diversas características del estudiante y de su familia, encontrando que hay varios factores explicativos del rendimiento académico: el 
género (las mujeres obtienen un mejor desempeño); la edad de ingreso (mejor desempeño de los más jóvenes); la educación de los padres (mientras más educado el padre, mejor el rendimiento); las horas trabajadas por parte del estudiante y el desempeño en la secundaria.

Garbanzo (2007) realizó una investigación sobre los factores asociados al rendimiento académico en estudiantes universitarios, en la cual indica que estos factores se agrupan en tres categorías: determinantes personales, institucionales y sociales.

Moreira (2009) realizó una investigación para identificar las variable exógenas y endógenas asociadas al rendimiento en la prueba de Matemáticas de bachillerato en educación media del sistema educativo formal de Costa Rica. Se concluyó que las variables exógenas que se encuentran asociadas al rendimiento son: la escolaridad de la madre, la condición de repitente y de aplazamiento en algún nivel de la secundaria, en Matemáticas.

Tonconi (2010) realizó una investigación sobre el análisis de los factores del rendimiento académico y la deserción de los estudiantes de la Facultad de Ingeniería Económica de la Universidad Nacional del Altiplano de Puno. Para la estimación del modelo sobre el rendimiento académico, utilizó el modelo econométrico lineal mediante mínimos cuadrados ordinarios, el cual indica que el rendimiento académico del estudiante es explicado significativamente en términos marginales por las variables como el número de créditos en que se matricula, el número de horas dedicadas al estudio por día, la asistencia a clases, el número de cursos que desaprobó, el ingreso económico mensual del estudiante, el tamaño familiar y el nivel de educación superior del jefe del hogar.

\section{Metodología}

Los datos utilizados en este estudio corresponden a los promedios finales de todas las evaluaciones de los estudiantes que cursaron el ciclo 2011-I de todos los cursos ofrecidos por el CEPRE-UNALM. El tamaño de la muestra aleatoria es de 320 estudiantes. Se realizó un muestreo sistemático de 20 estudiantes de cada una de las 16 aulas del CEPRE-UNALM. De cada aula se realizó una muestra aleatoria de 20 estudiantes de acuerdo con una razón constante que es igual al número de estudiantes entre 20, el primer estudiante de cada aula se escoge al azar y los 19 restantes de acuerdo a la razón constante de la lista de estudiantes del aula. Para el análisis de los factores que explican las diferencias en el rendimiento de los estudiantes, se consideraron las siguientes variables.

\section{Factores referidos al estudiante}

Lugar de procedencia. Esta variable se refiere al lugar de procedencia del estudiante. Se recogió a través de un ítem con dos alternativas: el estudiante es de Lima y el estudiante es de provincia.

Género. Esta variable recoge la información sobre el género del estudiante; se indagó a través de un ítem con dos alternativas: femenino y masculino.
Número de ciclos en el CEPRE-UNALM. Esta variable acopia información sobre el número de ciclos que el estudiante ha seguido en el CEPRE-UNALM. La variable se recogió a través de un ítem con dos alternativas: primer ciclo en el CEPRE-UNALM y más de un ciclo en el CEPRE-UNALM.

Colegio de procedencia. Las instituciones educativas pueden ser de gestión estatal o de gestión privada. Las primeras son creadas y sostenidas por el Estado. Las últimas son las creadas y administradas por personas de derecho privado, naturales o jurídicas. Esta variable recoge la información sobre el colegio de procedencia del estudiante y se indagó a través de un ítem con dos alternativas: el estudiante es de colegio estatal y el estudiante es de colegio privado.

Tipo de matrícula en el CEPRE-UNALM. Esta variable se refiere a la información acerca de la condición de becado o no becado del estudiante en el CEPREUNALM. La variable se recogió a través de un ítem con dos alternativas: el estudiante es becado en el CEPREUNALM y el estudiante no es becado en el CEPREUNALM.

\section{Factores referidos al aula}

Número de estudiantes por aula. Esta variable brinda información acerca de la cantidad de estudiantes por aula del CEPRE-UNALM.

Turno de clases en el CEPRE-UNALM. Esta variable reúne los datos sobre el turno de clases del estudiante. Se recogió a través de un ítem con dos alternativas: mañana $\mathrm{y}$ tarde.

Tipo de profesor en el aula. Esta variable recoge la información sobre si el estudiante tiene como profesor de aula, a un profesor de la UNALM o a un profesor que no es de la UNALM. Se estableció un ítem con dos alternativas para su recojo: el profesor de aula es de la UNALM y el profesor de aula no es de la UNALM.

Los modelos multinivel son ampliaciones de los modelos de regresión lineal clásicos, de tal forma que en realidad son varios modelos lineales para cada nivel. Así, los modelos de primer nivel están relacionados con uno de segundo nivel en el que los coeficientes de regresión del nivel 1 se regresan en un segundo nivel de variables explicativas y así sucesivamente para los diferentes niveles.

El análisis multinivel analiza datos que tienen una estructura jerárquica y nos permite estimar sin sesgos el efecto de variables contextuales e individuales. Los modelos multinivel facilitan la interpretación de la variabilidad observada en los resultados alcanzados en el promedio final en cada uno de los nueve cursos por un conjunto de $\boldsymbol{i}$ estudiantes, agrupados en $\boldsymbol{j}$ aulas del CEPRE-UNALM. En general, se espera que la 
variabilidad de los puntajes que miden el resultado del aprendizaje de los estudiantes agrupados dentro de una misma aula, presenten un cierto grado de homogeneidad, mientras que la mayor variabilidad se espera encontrar entre aulas. En este caso se tendrán dos niveles: el primer nivel es el estudiante y el segundo nivel es el aula.

El punto de partida del análisis multinivel es un modelo incondicional o modelo vacío o nulo que permite estimar la media global del rendimiento y calcular la proporción de las diferencias en rendimiento explicada por las características de los estudiantes y aquella explicada por los factores relacionados con el aula. El modelo nulo está dado por las siguientes ecuaciones:

Nivel 1:

$$
y_{i j}=\beta_{0 j}+\varepsilon_{i j}
$$

Donde $y_{i j}$ es la nota final que tiene un estudiante i en el aula j,

$\beta_{0 j}$ es el promedio de las notas finales del aula j-ésima y

$\varepsilon_{i j}$ es el efecto aleatorio asociado al i-ésimo estudiante en el aula $\mathrm{j}$ y se distribuye normalmente con media cero y varianza igual a $\sigma^{2}$.

Nivel 2: $\beta_{0 j}=\beta_{0}+u_{0 j}$

Donde $\beta_{0} \quad$ representa el gran promedio de las notas finales de la CEPRE - UNALM y

$u_{0 j}$ es el efecto aleatorio asociado a la j-ésima aula y se distribuye normalmente con media cero y una varianza igual a. $\boldsymbol{\tau}_{00}$

De la ecuación: $y_{i j}=\beta_{0}+u_{0 j}+\varepsilon_{i j}$, se halla que la varianza del rendimiento de los estudiantes es:

$\operatorname{Var}\left(y_{i j}\right)=\operatorname{Var}\left(\beta_{0}+u_{0 j}+\varepsilon_{i j}\right)=\tau_{00}+\sigma^{2}$

La varianza del rendimiento de los estudiantes puede ser descompuesta en dos partes: la primera recoge la variabilidad entre las aulas, mientras que la segunda recoge la variabilidad que existe dentro de las aulas. El análisis estadístico se realizó con el software MLwiN (Multilevel Modelling wi Nanostat) versión 2.23 que fue desarrollado desde 1980, primero como un comando de un programa basado en DOS, MLn, y desde 1998 como una versión Windows. Este software es producido por el proyecto de Modelos Multinivel en el Instituto de Educación, de la Universidad de Londres.

\section{Resultados y discusión}

A continuación, se mostrarán los resultados descriptivos de las variables estudiadas.

Las Tablas de la 2 hasta la 7 son de la muestra.
Tabla 1. Número de estudiantes por aula

\begin{tabular}{cc}
\hline Aula & Cantidad \\
\hline A & 57 \\
B & 59 \\
C & 45 \\
D & 44 \\
E & 56 \\
F & 54 \\
G & 55 \\
H & 55 \\
I & 53 \\
J & 52 \\
K & 50 \\
L & 48 \\
M & 58 \\
N & 59 \\
O & 56 \\
P & 61 \\
Total & 862 \\
\hline
\end{tabular}

Tabla 2. Colegio de procedencia

\begin{tabular}{cc}
\hline Tipo de colegio & Número de estudiantes \\
\hline Estatal & 154 \\
Privado & 166 \\
\hline Total & 320 \\
\hline
\end{tabular}

Tabla 3.Turnos de clases en el CEPRE-UNALM

\begin{tabular}{cc}
\hline Turno & Número de estudiantes \\
\hline Mañana & 240 \\
Tarde & 80 \\
\hline Total & 320 \\
\hline
\end{tabular}

Tabla 4. Género

\begin{tabular}{cc}
\hline Género & Número de estudiantes \\
\hline Femenino & 167 \\
Masculino & 153 \\
\hline Total & 320 \\
\hline
\end{tabular}

Tabla 5. Lugar de procedencia

\begin{tabular}{cc}
\hline Lugar & Número de estudiantes \\
\hline Lima & 263 \\
Provincia & 57 \\
\hline Total & 320 \\
\hline
\end{tabular}

Tabla 6. Tipo de matrícula en el CEPRE-UNALM

\begin{tabular}{cc}
\hline Tipo de matricula & Número de estudiantes \\
\hline Becado & 80 \\
No Becado & 240 \\
\hline Total & 320 \\
\hline
\end{tabular}


Tabla 7. Número de ciclos en el CEPRE-UNALM

\begin{tabular}{cc}
\hline Número de ciclos & Número de estudiantes \\
\hline Un ciclo & 225 \\
Más de un ciclo & 95 \\
\hline Total & 320 \\
\hline
\end{tabular}

Tabla 8. Tipo de profesor en el aula

\begin{tabular}{ccc}
\hline Cursos & Profesor de la UNALM & Profesor no UNALM \\
\hline Algebra & 1 & 5 \\
Aritmética & 3 & 4 \\
Geometría & 2 & 4 \\
Física & 2 & 5 \\
Química & 4 & 2 \\
Biología & 2 & 3 \\
Trigonometría & 2 & 5 \\
Razonamiento Matemático & 0 & 6 \\
Razonamiento Verbal & 1 & 4
\end{tabular}

Tabla 9. Modelo vacío o nulo para cada uno de los cursos que desarrolla la CEPRE-UNALM

\begin{tabular}{|c|c|c|c|c|c|c|c|c|c|}
\hline & Geometría & Algebra & Química & Biología & Física & Aritmética & Trigonometría & $\begin{array}{l}\text { Raz. } \\
\text { Verbal }\end{array}$ & $\begin{array}{c}\text { Raz. } \\
\text { Matemático }\end{array}$ \\
\hline Intercepto & 7,865 & 8,139 & 8,750 & 8,203 & 7,327 & 8,529 & 9,1 & 8,772 & 7,356 \\
\hline Entre aulas & 16,905 & 16,771 & 17,903 & 11,883 & 16,648 & 13,560 & 21,425 & 2,668 & 9,286 \\
\hline Entre estudiantes & 17,060 & 16,632 & 17,823 & 15,538 & 14,688 & 18,521 & 21,735 & 14,192 & 11,331 \\
\hline $\begin{array}{l}\text { Porcentaje de la } \\
\text { varianza atribuida a la } \\
\text { diferencia entre aulas }\end{array}$ & 49,7 & 50,2 & 50,1 & 43,3 & 53,1 & 42,3 & 49,6 & 15,8 & 45 \\
\hline $\begin{array}{l}\text { Porcentaje de la } \\
\text { varianza atribuida a } \\
\text { la diferencia entre } \\
\text { estudiantes }\end{array}$ & 50,3 & 49,8 & 49,9 & 56,7 & 46,9 & 57,7 & 50,4 & 84,2 & 55 \\
\hline
\end{tabular}

Tabla10. Modelo final de factores asociados al rendimiento en cada uno de los cursos que desarrolla la CEPREUNALM

\begin{tabular}{|c|c|c|c|c|c|c|c|c|c|}
\hline & Geometría & Algebra & Química & Biología & Física & Aritmética & Trigonometría & $\begin{array}{l}\text { Raz. } \\
\text { Verbal }\end{array}$ & $\begin{array}{c}\text { Raz. } \\
\text { Matemático }\end{array}$ \\
\hline Intercepto & $11,153 * *$ & $10,732 * *$ & $9,899 * *$ & $9,692 * *$ & $8,276 * *$ & $8,037 * *$ & $11,120 * *$ & $6,43 * *$ & $6,566 * *$ \\
\hline Género & $0,739 *$ & 0,472 & 0,194 & $-0,220$ & 0,999 & 0,189 & 0,674 & $-0,232$ & 0,307 \\
\hline $\begin{array}{l}\text { Tipo de matrícula } \\
\text { en el CEPRE- } \\
\text { UNALM }\end{array}$ & $-6,787 * *$ & $-6,849 * *$ & $-6,355 * *$ & $-4,963 * *$ & $-6,171 * *$ & $-6,478 * *$ & $-7,057 * *$ & $-3,180 * *$ & $-4,947 * *$ \\
\hline $\begin{array}{l}\text { Número de ciclos } \\
\text { en el CEPRE- } \\
\text { UNALM }\end{array}$ & $1,949 * *$ & $1,867 * *$ & $2,524 * *$ & $2,452 * *$ & $1,370 * *$ & $1,890 * *$ & $3,143 * *$ & 0,543 & $1,704 * *$ \\
\hline $\begin{array}{l}\text { Lugar de } \\
\text { procedencia }\end{array}$ & $-0,155$ & 0,275 & 0,182 & 0,232 & 0,117 & 0,123 & $-0,009$ & 0,253 & 0,187 \\
\hline $\begin{array}{l}\text { Turno de clases } \\
\text { en el CEPRE- } \\
\text { UNALM }\end{array}$ & 0,147 & 0,112 & $-0,401$ & 0,029 & $1,038 *$ & 0,293 & 0,117 & 0,124 & 0,210 \\
\hline $\begin{array}{l}\text { Colegio de } \\
\text { procedencia }\end{array}$ & 0,276 & 0,511 & $-0,002$ & 0,286 & 0,128 & $0,962 *$ & 0,457 & $0,791^{*}$ & $0,959 * *$ \\
\hline Tamaño del aula & $-0,004$ & $-0,001$ & 0,022 & 0,010 & 0,007 & 0,056 & 0,008 & 0,070 & 0,043 \\
\hline $\begin{array}{l}\text { Tipo de profesor en } \\
\text { el aula }\end{array}$ & $-0,215$ & $0,805 *$ & $0,968 *$ & $-0,883 *$ & $2,132 * *$ & 0,075 & $-0,176$ & 0,025 & \\
\hline
\end{tabular}

* Significativa al $5 \%$ 
Los resultados del modelo multinivel para cada uno de los cursos figuran en las Tablas 9 y 10. En la Tabla 9 se presenta el modelo nulo o vacío para cada uno de los cursos que se desarrolla en el CEPRE-UNALM y en la Tabla 10 se presenta el modelo final para cada uno de los cursos.

Geometría. A partir del modelo nulo se obtuvo como resultado que el $49,7 \%$ de las diferencias en rendimiento en Geometría se daba entre aulas y el 50,3\% entre estudiantes. Los resultados del modelo final muestran que, después de controlar todos los factores, es el tipo de matrícula la que tiene un mayor efecto sobre el rendimiento; es decir, el rendimiento promedio de un estudiante se vería reducido en aproximadamente 6,787 puntos si es que este fuera no becado. Asimismo, se observa que el rendimiento promedio de un estudiante se vería aumentado en aproximadamente 2,688 puntos si es que este fuera masculino y estuviera estudiando más de un ciclo en el CEPRE-UNALM. El resto de variables no resultaron significativas.

\begin{abstract}
Algebra. A partir del modelo nulo se obtuvo como resultado que el $50,2 \%$ de las diferencias en rendimiento del curso se daba entre aulas y el 49,8\% entre estudiantes. Los resultados del modelo final muestran que, después de controlar todos los factores, es el tipo de matrícula la que tiene un mayor efecto sobre el rendimiento; es decir, el rendimiento promedio de un estudiante se vería reducido en aproximadamente 6,849 puntos si es que este fuera no becado. Asimismo, se observa que el rendimiento promedio de un estudiante se vería aumentado en aproximadamente 2,672 puntos si es que su profesor es de la UNALM y estuviera estudiando más de un ciclo en el CEPRE-UNALM. El resto de variables no resultaron significativas.
\end{abstract}

Química. A partir del modelo nulo se obtuvo como resultado que el $50,1 \%$ de las diferencias en rendimiento en Química se daba entre aulas y el 49,9\% entre estudiantes. Los resultados del modelo final muestran que, después de controlar todos los factores, es el tipo de matrícula la que tiene un mayor efecto sobre el rendimiento en este curso; es decir, el rendimiento promedio de un estudiante se vería reducido en aproximadamente 6,355 puntos si es que este fuera no becado. Asimismo, se observa que el rendimiento promedio de un estudiante se vería aumentado en aproximadamente 0,968 puntos si su profesor no es de la UNALM. El resto de variables no resultaron significativas.

Biología. A partir del modelo nulo se obtuvo como resultado que el $43,3 \%$ de las diferencias en rendimiento en Biología se daba entre aulas y el 56,7\% entre estudiantes. Los resultados del modelo final muestran que, después de controlar todos los factores, es el tipo de matrícula la que tiene un mayor efecto sobre el rendimiento en este curso; es decir, el rendimiento promedio de un estudiante se vería reducido en aproximadamente 5,846 puntos si es que este fuera no becado y su profesor fuera de la UNALM. Asimismo, se observa que el rendimiento promedio de un estudiante se vería aumentado en aproximadamente 2,452 puntos si es que este estuviera estudiando más de un ciclo en el CEPRE-UNALM. El resto de variables no resultaron significativas.

Física. A partir del modelo nulo se obtuvo como resultado que el $53,1 \%$ de las diferencias en rendimiento en Física se daba entre aulas y el 46,9\% entre estudiantes. Los resultados del modelo final muestran que, después de controlar todos los factores, es el tipo de matrícula la que tiene un mayor efecto sobre el rendimiento en este curso; es decir, el rendimiento promedio de un estudiante se vería reducido en aproximadamente 6,171 puntos si es que este fuera no becado. Asimismo, se observa que el rendimiento promedio de un estudiante se vería aumentado en aproximadamente 4,54 puntos si es que este estuviera estudiando más de un ciclo en el CEPREUNALM, su turno de clases sería tarde y su profesor sería de la UNALM. El resto de variables no resultaron significativas.

Aritmética. A partir del modelo nulo se obtuvo como resultado que el $42,3 \%$ de las diferencias en rendimiento en Aritmética se daba entre aulas y el 57,7\% entre estudiantes. Los resultados del modelo final muestran que, después de controlar todos los factores, es el tipo de matrícula la que tiene un mayor efecto sobre el rendimiento en este curso; es decir, el rendimiento promedio de un estudiante se vería reducido en aproximadamente 6,478 puntos si es que este fuera no becado. Asimismo, se observa que el rendimiento promedio de un estudiante se vería aumentado en aproximadamente 2,852 puntos si es que este estuviera estudiando más de un ciclo en el CEPRE-UNALM y procediera de un colegio privado. El resto de variables no resultaron significativas.

Trigonometría. A partir del modelo nulo se obtuvo como resultado que el $49,6 \%$ de las diferencias en rendimiento en Trigonometría se daba entre aulas y el 50,4\% entre estudiantes. Los resultados del modelo final muestran que, después de controlar todos los factores, es el tipo de matrícula la que tiene un mayor efecto sobre el rendimiento en este curso; es decir, el rendimiento promedio de un estudiante se vería reducido en aproximadamente 7,057 puntos si es que este fuera no becado. Asimismo, se observa que el rendimiento promedio de un estudiante se vería aumentado en aproximadamente 3,143 puntos si es que este estuviera estudiando más de un ciclo en el CEPRE-UNALM. El resto de variables no resultaron significativas. 
Razonamiento Verbal. A partir del modelo nulo se obtuvo como resultado que el $15,8 \%$ de las diferencias en rendimiento en Razonamiento Verbal se daba entre aulas y el 84,2 \% entre estudiantes. Los resultados del modelo final muestran que, después de controlar todos los factores, es el tipo de matrícula la que tiene un mayor efecto sobre el rendimiento en este curso; es decir, el rendimiento promedio de un estudiante se vería reducido en aproximadamente 3,18 puntos si es que este fuera no becado. Asimismo, se observa que el rendimiento promedio de un estudiante se vería aumentado en aproximadamente 0,791 puntos si es que este procediera de un colegio privado. El resto de variables no resultaron significativas.

Razonamiento Matemático. A partir del modelo nulo se obtuvo como resultado que el $45 \%$ de las diferencias en rendimiento en Razonamiento Matemático se daba entre aulas y el $55 \%$ entre estudiantes. Los resultados del modelo final muestran que, después de controlar todos los factores, es el tipo de matrícula la que tiene un mayor efecto sobre el rendimiento en este curso; es decir, el rendimiento promedio de un estudiante se vería reducido en aproximadamente 4,947 puntos si es que este fuera no becado. Asimismo, se observa que el rendimiento promedio de un estudiante se vería aumentado en aproximadamente 2,663 puntos si es que este estuviera estudiando más de un ciclo en el CEPRE-UNALM y procediera de un colegio privado. El resto de variables no resultaron significativas. Ningún profesor de Razonamiento Matemático es de la UNALM.

En primer lugar, en el modelo nulo de todos los cursos, menos en Razonamiento Verbal, se observa que la mitad de la variabilidad del rendimiento se debe a las características de las aulas y la otra mitad se debe a las características de los estudiantes. Esto significa que se pueden implementar medidas de política orientadas al aula, como por ejemplo mejorar las estrategias de enseñanza de los docentes, el trato a los estudiantes, etc. En Razonamiento Verbal la variabilidad de su rendimiento se debe más a las características de los estudiantes.

En el curso de Geometría los estudiantes varones muestran un mejor rendimiento, pero en los demás cursos de Matemáticas (Álgebra, Aritmética, Razonamiento Matemático y Trigonometría) y en Razonamiento Verbal el género no es estadísticamente significativo. En otras investigaciones realizadas, se encontró que los estudiantes varones, tendencialmente, presentan mejores resultados en Matemática, mientras que las estudiantes mujeres tienden a obtener un mejor rendimiento en Comunicación. Cabe señalar que si bien algunos estudios han tratado de mostrar que dichas diferencias están relacionadas con las características biológicas entre ambos sexos (Kimura, 2004), otros estudios han demostrado que estas diferencias se explicarían más bien por las diferencias en las prácticas de socialización de mujeres y varones, que favorecen el desarrollo de un determinado tipo de habilidades según el género, así como a su correlato en las percepciones que tienen los docentes sobre las habilidades que pueden desarrollar los estudiantes (Shakeshaft, 1995; Shepardson y Pizzini, 1992).

Antes de iniciar el ciclo académico en el CEPRE UNALM, se convoca a un concurso de concesión de becas y los estudiantes que se hacen acreedores a las mismas ya vienen con una preparación preuniversitaria, mientras los no becados, en su mayoría, son recién egresados de la secundaria; es por esta razón que el rendimiento de los becados es mejor.

Los estudiantes que tienen más de un ciclo en el CEPRE - UNALM incrementan su rendimiento. Esto significa que el Centro les brinda las condiciones para que el estudiante logre su aprendizaje. Los estudiantes de colegios privados presentan mejor rendimiento que los estudiantes de colegios estatales en los cursos de Aritmética, Razonamiento Matemático y Razonamiento Verbal; esta diferencia se puede deber a que los colegios estatales atienden a la población económicamente más desfavorecidas, al tiempo de dedicación que le brindan a estos cursos y a los contenidos que desarrollan en cada uno de estos. En los colegios privados cada curso tiene un profesor, en cambio en los colegios estatales no siempre es así.

En el CEPRE, hay dos tipos de profesores: el profesor que es de la UNALM y el que no es de la UNALM. Se ha encontrado una asociación de esta variable con el rendimiento, en los cursos de Física, Algebra, Química y Biología, que puede tener un efecto negativo o positivo en este. Al respecto, se tendría que analizar qué características de los docentes se relacionan con el buen desempeño de los estudiantes.

\section{Conclusiones}

El tipo de matrícula en el CEPRE-UNALM se encuentra asociado al rendimiento de todos los cursos que se desarrollan en el CEPRE-UNALM; en este caso, el rendimiento promedio de un estudiante en cualquiera de los cursos se vería reducido si el estudiante es no becado.

El género se encuentra asociado al rendimiento en Geometría. Es el único curso en la cual resultó significativa la variable género. Si el estudiante es masculino, su rendimiento promedio se vería aumentado en 0,739 puntos. En otras investigaciones este factor resulta asociado al rendimiento en matemáticas.

El número de ciclos en el CEPRE-UNALM se encuentra asociado al rendimiento de todos los cursos, menos el curso de Razonamiento Verbal. Resulta que el rendimiento promedio de un estudiante se vería incrementada si este tiene más de un ciclo en el CEPRE-UNALM.

El colegio de procedencia se encuentra asociado al rendimiento de los cursos de Aritmética, Razonamiento Verbal y Razonamiento Matemático. En este caso el rendimiento promedio de un estudiante se vería incrementado si este procediera de un colegio privado. 
El tipo de profesor en el aula se encuentra vinculado al rendimiento de los cursos de Física, Algebra, Química y Biología. El rendimiento promedio de un estudiante en Física y Algebra se vería incrementado si su profesor es de la UNALM, en Biología se vería disminuida si su profesor es de la UNALM y en Química se vería incrementado si su profesor no es de UNALM.

Se recomienda ampliar el trabajo de investigación para determinar el efecto que tiene la habilidad, nivel de satisfacción y expectativas del docente, relación profesor - estudiante, interés del estudiante por un curso determinado, antecedentes socioeconómicos, culturales y académicos de los estudiantes, materiales de estudio, asesorías a cargo de los tutores, apoyo psicológico.

\section{Literatura citada}

Bowles, S. and Levin, H. 1968. The determinants of Scholastic Achievement- An Appraisal of Some Recent Evidence. Journal of Human Resources, 3(1): 3-24.

Coleman, J.; Campbell, E.; Hobson, C.; McPartland, J.; Mood, A.; Weinfall, F. and York, R. 1966. Equality of Educational Opportunity. Washington DC: Department of Health, Education, and Welfare.

Carrasco, J. 1985. La recuperación educativa. España: Editorial Anaya.

Heran y Villarroel. 1987. Caracterización de algunos factores del alumno y su familia de escuelas urbanas y su incidencia en el Rendimiento de Castellano y Matemática en el primer ciclo de Enseñanza General Básica. Editado por CPEIP.

Porto y Di Gresia. 2004. Revista de Economía y Estadística. Vol. XLII, pp. 93-113.

Instituto de Economía y Finanzas - Facultad de Ciencias Económicas. Argentina: Universidad Nacional de Córdoba.

Pizarro, R. 1985. Rasgos y actitudes del profesor efectivo. (Tesis de grado). Pontificia Universidad Católica de Chile. Santiago, Chile.

Tonconi Quispe, J. 2010. Factores que influyen en el rendimiento académico y la deserción de los estudiantes de la Facultad de Ingeniería Económica de la UNAPUNO, periodo 2009. Revista Académica Cuadernos de Educación y Desarrollo, 2 (11).

Ministerio de Educación del Perú. 2004. Unidad de Medición de la Calidad Educativa 2004. Factores asociados al rendimiento estudiantil. Resultados de la Evaluación Nacional 2001. Documento de trabajo. 